\title{
Is the Near-Death Experience Only N-Methyl-D-Aspartate Blocking?
}

\author{
Peter Fenwick, M.D., F.R.C.Psych. \\ Institute of Psychiatry, London, England
}

\begin{abstract}
Karl Jansen's interesting hypothesis that near-death experiences (NDEs) result from blockade of the N-methyl-D-aspartate receptor has several weaknesses. Some NDEs occur to individuals who are neither near death nor experiencing any event likely to upset cerebral physiology as Jansen proposed; thus his hypothesis applies only to a subset of NDEs that occur in catastrophic circumstances. For that subset, the clarity of NDEs and the clear memory for the experience afterward are inconsistent with compromised cerebral function. Jansen's analogy between NDEs and ketamine-induced hallucinations is weakened by the fact that most ketamine users do not believe the events they perceived really happened. Temporal lobe seizures do not resemble NDEs as Jansen postulated; they are confusional, rarely ecstatic, and never clear, as are NDEs, nor are they remembered afterward. Jansen's hypothesis assumes the standard scientific view that brain processes are entirely responsible for subjective experience; however, NDEs suggest that that concept of the mind may be too limited, and that in fact personal experience may continue beyond death of the brain.
\end{abstract}

Karl L. R. Jansen has proposed a hypothesis describing a mechanism that makes a major contribution to the understanding of the near-death experience (NDE). He suggested that the NDE is the result of the blocking of the phencyclidine (PCP) site on the N-methyl$\mathrm{D}$-aspartate (NMDA) receptor. This is an interesting hypothesis, and it is likely that the suggestion that the NMDA receptor is involved in a subset of NDEs is correct. However, Jansen's paper, like many before it, suffers from a number of weaknesses, some outlined by the

Peter Fenwick, M.D., F.R.C.Psych., is Consultant Neuropsychiatrist at the Bethlem Royal and Maudsley Hospitals and Senior Lecturer at the Institute of Psychiatry in London, England. Reprint requests should be addressed to Dr. Fenwick at the Institute of Psychiatry, deCrespigny Park Road, London S.E.5, United Kingdom. 
author. In reviewing this hypothesis, it is important to look at its implications from several different levels. When arguing for an explanation of the NDE, chemical, clinical, and philosophical aspects should all be included. I shall discuss these areas independently.

Jansen started his paper with a philosophical statement concerning death and the nature of mind. This point requires answering, but for the first part of this article I would like to take the position, suggested by Jansen, that mind arises from neuronal activity, and that consciousness is local to brain processes.

The first point is: how do we define an NDE? Are we to consider only those NDEs that occur with catastrophic brain failure, or should we include those NDEs that occur in different circumstances? Jansen did not suggest that his theory is applicable to all cases of NDEs, but argued that it can explain most experiences, put generally, because the PCP receptor is involved in perception.

The full breadth of near-death phenomena came to my attention after a television program and publication of several articles in the United Kingdom. The British branch of the International Association for Near-Death Studies (IANDS-UK) and I have received more than 2,000 letters containing experiences. From these letters we selected a subset of experiences that we felt were most typical of the core NDE. We mailed 500 questionnaires to this group of people and received over 370 replies (Fenwick and Fenwick, 1995). The selection of this data set was biased by several important factors. First, the group was self-selected. Second, the people who wrote were those who were sufficiently interested in their experiences to want to communicate something that was important to them. This adds a significant selection bias, in that people who have had negative or neutral experiences are much less likely to want to communicate them than those who have had positive experiences. However, accepting these deficiencies, these NDEs were reported as having occurred under many different situations, some of which were not near death, nor accompanied by any threatening event likely to have upset cerebral physiology in the way proposed by Jansen's hypothesis.

Experiences were reported to have occurred when experiencers were awake and relaxed, when they were depressed, and in minor infections and in routine anesthesia. We rejected those that were reported to have occurred in sleep. The largest number certainly occurred in catastrophic circumstances. Our sample makes the point that NDEs judged entirely by their phenomenology occur in many different circumstances; thus any theory that links the NDE only to 
brain catastrophe or to a special brain physiological mechanism must provide only a partial and limited explanation. Elizabeth Fenwick and I (Fenwick and Fenwick, 1995) have suggested that a detailed look at the phenomenology of the NDE allocates it to a group of experiences that is already well studied and understood, that of the mystical experience. This explanation has the advantage that mystical experiences too have multiple causes. The hypothesis put forward by Jansen should thus be limited to a subset of NDEs that occur in catastrophic circumstances.

Jansen argued, correctly in my view, that a clear sensorium and a feeling of absolute reality do not negate the suggestion that these experiences are hallucinations. From a scientific point of view it is clear that the majority of the NDEs must be hallucination, if one excludes those out-of-body experiences for which a veridical nature is claimed, as the world described is private to the individual and not held in common between subjects. The fact that we define the NDE mainly as an hallucination is of little help in terms either of the experience's likely genesis or of its philosophical explanation. It simply raises questions about the subjective nature of mind. It does, however, help to direct attention, as Jansen has done, to other situations in which hallucinations that have a similar form occur.

I was interested in the comment that only 30 percent of normal subjects given ketamine were certain that the events had really occurred. In NDEs this percentage is much higher, and this point weakens a ketamine-like effect as the only explanation. However, in defense of the ketamine hypothesis, it could be that those people given ketamine would naturally tend to attribute whatever experience they had to the drug, and so would be less likely to regard it as "real."

The phenomenon of a clear sensorium in catastrophic brain states is more difficult to explain. Any physician dealing with head injury, epilepsy, or altered cerebral physiology knows that as cerebral function becomes compromised it becomes disorganized. Even in such simple circumstances as ordinary fainting, recovery from the faint is recovery from a confusional process. Acute cerebral catastrophes result in confusion and not clarity. This important fact is overlooked by those attributing simple chemical explanations to the NDE. Although ketamine may produce experiences that are similar to the NDE, and Jansen has argued cogently that it does, he does not explain how these same experiences can arise in a dysfunctional brain. His argument is that when brain processes have been so disorganized 
that there is loss of consciousness, consciousness can then be resynthesized in its clarity by a brain mechanism such as flooding the brain with NMDA inhibition. Surely the very fact that consciousness has been lost would argue that cortical activity is insufficient to sustain high quality and clear consciousness as would be required for an NDE.

The only way round this dilemma is to argue that the experience arises as consciousness is being recovered or lost, when cerebral function is still sufficiently intact to maintain coherent and clear cerebral experience. There are arguments, mainly related to memory, that I will discuss further below, which make it unlikely that many NDEs could occur as consciousness is being lost. The alternative conclusion would therefore have to be that NDEs occur with the return of the cerebral processing involved in ordinary conscious experience. However, the difficulty here is that recovery from a cerebral catastrophe is via a confusional state and it thus seems unlikely that an arousal through confusion could produce both the clarity of the experience and the confusion of arousal.

In our series there were some specific accounts that made this point even more starkly. We had patients who were head-injured and whose arousal was confusional and showed all the characteristics and mental states that would be expected after a severe head injury. Yet within this dense confusional state, but attributed by the individual to the time of unconsciousness, was full memory of a wonderfully clear NDE. It is worth noting that in severe head injury memory for the accident and for the confusional awakening in hospital is absent, and this was so in our cases of head injury. Except by special pleading, it is not possible with our current understanding of cerebral functioning to explain, on a simple chemical theory, how, within dense unconsciousness and with absence of memory, the brain can structure and remember a clear comprehensive experience. This is an interesting point and is a challenge to our current understanding of brain function (Cartlidge, 1991; Teasdale, 1991).

One of the most puzzling features of NDEs, besides their clarity in the presence of cerebral catastrophe, is the clear memory for the experience. As mentioned above, memory is very sensitive to brain injury, and length of amnesia before and after unconsciousness is a way of determining the severity of the injury. It is thus unlikely that cerebral events occurring during this period of total amnesia would ordinarily be remembered (Cartlidge, 1991; Teasdale 1991). 
Jansen described the excitatory and inhibitory properties of ketamine and decided on balance against it being excitatory, though it is probably neuroprotective. He concluded that it is likely to be inhibitory. This leads to a discussion of the role of epilepsy in NDEs. There is much about the possible role of epilepsy, abnormal temporal lobe functioning, and abnormal hippocampal functioning in the NDE literature (Saavedra-Aguilar and Gómez-Jeria, 1989), which Jansen, quite rightly, wanted to include in his theory. In my view much of the discussion of temporal lobe epileptic (TLE) activity as a component of the NDE argues without the data to support it.

Let's start with temporal lobe seizures. We can divide these into those starting in the lateral temporal cortex and those arising from the hippocampus or the amygdala, the medial temporal structures. Let's consider those arising from the hippocampus or the amygdala. The international definition of these seizures is partial complex seizures; they are termed "complex" because they lead to an alteration of consciousness. This alteration in consciousness is confusional. When patients have seizures originating in the medial temporal structures they usually have a disorder of consciousness, and the mental phenomena are usually those of fear or extreme fear.

Positive auras and feelings, so common in the NDE, are reported in only a very small minority of medial temporal seizures. In William Gowers' (1881) study of 505 epileptic auras only 3 percent were said to be emotional and none positive. In William Lennox's (1960) study of 1,017 auras, only 9 were said to be pleasant ( 0.9 percent), and of these "only a few showed positive pleasure." Wilder Penfield and Kristian Kristiansen (1951) cited only one case with an aura of a pleasant sensation and it was followed by an epigastric feeling of discomfort. Until 1980, no cases of temporal lobe epilepsy and an ecstatic aura had been reported (Cirignotta, Todesco, and Lugaresi, 1980). Despite this, ecstatic states were frequently attributed in the literature to TLE. As Henri Gastaut (1978) explained, this was because people expected ecstatic states to be present (Fenwick, 1983).

Thus the phenomenology of discharges in this brain area bears no resemblance to that of the NDE. More importantly, when the seizures spread out from the medial temporal structures into the temporal cortex more complex phenomena may arise, but these are always confusional and never complex and clear like the NDE. It is thus unlikely that any alterations in electrical activity of a seizure type arising in the medial temporal structures could contribute to the NDE. 
Those seizures arising from the lateral temporal cortex do contain more complex phenomena. There may be alterations in space and time and body image. Déjà vu experiences can also arise, suggesting the false attribution of meaning to current experiences. But again these seizures are partial complex seizures and confusional. Thus abnormal discharges in the temporal lobe may produce confusional fragments of phenomena sometimes seen in NDEs (Williamson, Wieser, and Delgado-Escueta, 1987). This is a very long way from arguing that seizure discharges in these areas, resulting from brain catastrophe, can give rise to the clearly remembered, highly structured NDE. Finally, seizure discharges that involve or spread into the medial temporal structures lead to abolition of memory for what occurred during the seizure, and afterwards in the automatism, if one occurs. Many patients with prolonged automatisms have no memory for the complex events that occur during their wanderings. Thus even if temporal lobe seizures did contribute to the NDE the experiences would not be remembered. As yet I do not think there is sufficient evidence to support the idea of seizure discharges in the temporal lobe being responsible for the NDE.

Quite clearly, drug experiences, which mobilize widely different brain areas in addition to the temporal lobe, can produce higher order experiences that also include some of these same fragments of temporal lobe experience seen in temporal lobe seizure discharges. This is, however, not to argue that temporal lobe seizure discharges lead in any sense to these wider coherent experiences. It must also be recognized that epilepsy surgery units involved in temporal lobe surgery routinely implant indwelling cerebral electrodes either into the hippocampus and amygdala or beside the hippocampus and amygdala (foramen ovale electrodes); these electrodes usually show continuous or frequently abnormal electrical activity. Epileptic spike discharges are common. Yet none of these discharges are correlated with NDE-like phenomena. Indeed, unless the individual epileptic discharges spread to form either local or more general seizure discharges, there is little effect on cognition, and the individual is unaware that they are occurring (Ojemann and Engel, 1987). Thus on both electrical grounds and clinical grounds, the argument that temporal lobe seizure-like activity is responsible for near-death phenomena must be discarded. There is one caveat: all the data so far described refer to patients with epilepsy and taking anticonvulsant drugs, so we do not know how the nonepileptic brain would respond, though it is unlikely to be very different. 
If one is to retain the idea of a change in hippocampal or hippocampal-amygdalal function during the NDE, one must argue specifically for very organized changes in functioning. Much more is now known about the function of the hippocampus. First, postmortem specimens resected from patients with temporal lobe epilepsy usually show the presence of bilateral hippocampal disease and hippocampal cell loss (Babb and Brown, 1987). It is thus possible to have major pathology within the hippocampus and for none of these experiences to arise. Clearly the demonstration of pathology does not necessarily negate the possibility that these structures mediate the NDE, but it does suggest that damage to these structures does not necessarily lead to an NDE, as was postulated in Jansen's article.

Further, there is now a large body of data showing the relationship between hippocampal damage and memory function. It seems likely that in right-handed people the left hippocampus is involved in verbal memory and the right hippocampus in visuospatial memory (Jones-Gotman, 1987). Damage to one hippocampus early in life may result in the other hippocampus taking over, to some extent, the function of the damaged side. Clearly, arguing that electrical discharges in the hippocampus give rise to the full panoply of phenomenology of the NDE is far too simplistic. However, something can be salvaged from the hippocampal theory. If it is postulated that the hippocampus is involved in the direct way Jansen suggested, then an interesting hypothesis can be generated as to the content of the memories that occur in the NDE. If one hippocampus is damaged, there should be an appropriate lack of recalled memories in the NDE, either visuospatial or verbal, depending on the side of the damage.

Turning now to the nature of mind, Jansen quoted the standard identity theory, which states that brain processes are entirely responsible for, and identical with, subjective experience. In other words, he presented the standard scientific view (Dennett, 1993). However, one of the interesting and exciting points about the NDE is that it suggests that our view of mind may be too limited. It raises the possibility that mind may be nonlocal. Nonlocal means that although brain processes within the skull are involved in the structuring of mind, the effects of mind may extend beyond the brain. If nonlocality is a possibility, we have then to ask what we mean by "mind."

For simplicity's sake I will define mind as "subjective experience," although I am aware of the limitation of this definition and the philosophical problems that arise by using it. Many scientifically valid parapsychological experiments have suggested that subjective expe- 
rience may be transmitted from brain to brain directly in telepathy, or influence physical matter outside the brain in psychokinesis (Bem and Honorton, 1994; Jahn and Dunne, 1987; Wolman, 1977). These data are not well known in scientific circles and are seldom quoted in scientific articles when subjective experience and brain function are discussed. The reason for this is that as yet we have no clear theory to explain paranormal experiences, and as such these phenomena are outside science. One of the challenges of the NDE is that it puts before us phenomena that could be explained much more easily by such a nonlocal theory of mind than by standard scientific thinking.

Out-of-body experiences are frequently reported to occur in the early part of the NDE. These out-of-body experiences have a heterogeneous phenomenology and some can be explained quite easily by conventional science, which suggests that the brain creates our subjective experience of our body and its position in space from sensory information. The body is experienced as being elsewhere, for example on the ceiling, either for psychological reasons or because an alteration in brain physiology, such as may occur in acute anxiety, transforms the coordinates of the body's position in subjective space. What the subject thinks and hears while apparently on the ceiling is simply the continuation of sensory flow into the brain allowing an altered construction of reality.

However, anecdotal cases have been reported that seem to indicate quite persuasively the involvement of some other mechanism and the occurrence of veridical out-of-body perceptions. The subject apparently experiences objects in the external world that could not be perceived by the senses. An example is the out-of-body experience during an NDE, when the subject may feel that he or she floats into a different room and hears a conversation that could not possibly have been heard otherwise. In this case, if the account is correct, data have to have been received directly by the mind/brain without intervention of the senses. There are numerous anecdotal accounts in the near-death literature of this kind of experience. It would be interesting to know if there are similar accounts by people who have had out-of-body experiences under ketamine, and if so, if there is any objective evidence that they are veridical.

Other newer, nonparapsychological theories have sought to explain nonlocality of mind by suggesting that quantum mechanical processes may take place in the brain. Stewart Hameroff (1987) and Roger Penrose (1994) argued for special energetic processes either in 
the cell (microtubules) or on the cell membrane (Frohlich oscillators or Bose-Einsten condensates) that would allow quantum mechanical effects to occur in the brain. Prior to these theories the brain had always been thought to be "too hot and too wet" for quantum mechanics to play a part in the way it operates. These are exciting new theories, as they would support the idea of nonlocality of mind.

Finally, although this was not the thrust of Jansen's article, it is worth a look at the philosophical statements at the beginning of his paper. There was an assumption that chemical and brain processes are all there is to mind; understand brain processes and mind is completely explained. Some of the phenomena of the NDE suggest that subjective experience may be maintained in the face of severe cerebral catastrophe. If this is a possibility, then the maintenance of subjective experience when brain function is severely disorganized has consequences for our understanding of brain processes and the nature of mind. It even raises the possibility that there might be a continuation of subjective experience after brain death.

This is an area of growing interest in philosophy. Our implicit everyday scientific assumptions in science do require questioning. Current science is based on the philosophical assumptions of Galileo, who argued for a two-stuff universe, matter and energy, and for this stuff to have primary and secondary qualities. Primary qualities are the objective qualities of the physical world, such as weight, velocity, and momentum. Secondary qualities are the qualities of subjective experience, such as smell, taste, love, and beauty. Galileo argued that secondary qualities were not in the domain of science; thus our current scientific theories are all based on an objective external world that contains no secondary qualities. And yet we insist on using these theories to try to explain these very qualities, which by definition cannot be explained by Galilean science.

More recently, Thomas Kuhn (1970) pointed out that what we think of as independent objective scientific facts are "value laden." By this he meant that scientific facts arise within a theory that itself is part of a culture, and thus our particular view is cultural, and so our objective facts are relative. Max Velmans (1991, 1993) maintained that an objective fact is a subjective cognition held in common between a group of people and verifiable by them, whereas a subjective cognition is private to the individual, does not have the quality of objective validation, but is no less true. With these limitations, it is clear that our science as currently formulated is not able to explain the implications of the NDE. Science is able to note brain correlates 
of the NDE where they exist, but it cannot argue from these correlates to the significance, or even the presence, of subjective experience. One is left only with the subjective accounts. Science, by definition, cannot explain subjective experience, so it can neither refute nor confirm the possibility that these subjective accounts do indeed suggest that some form of personal experience may continue during the unconsciousness of brain catastrophe or even after brain death.

\section{References}

Babb, T. L., and Brown, W. J. (1987). Pathological findings in epilepsy. In J. Engel (Ed.), Surgical treatment of the epilepsies (pp. 511-540). New York, NY: Raven Press.

Bem, D., and Honorton, C. (1994). Does psi exist? Replicable evidence for an anomalous process of information transfer. Psychological Bulletin, 115, 4-18.

Cartlidge, N. (1991). Head injury: Outcome and prognosis. In M. Swash and J. Oxbury (Eds.), Clinical neurology (pp. 699-707). Edinburgh, Scotland: Churchill Livingstone.

Cirignotta, F., Todesco, C. V., and Lugaresi, E. (1980). Temporal lobe epilepsy with ecstatic seizures (so-called Dostoevsky epilepsy). Epilepsia, 21, 705-710.

Dennett, D. (1991). Consciousness explained. Boston, MA: Little, Brown.

Fenwick, P. B. C. (1983). Some aspects of the physiology of the mystical experience. In J. Nicholson and B. Foss (Eds.), Psychology survey, No. 4 (pp. 203-223). Leicester, England: British Psychological Society.

Fenwick, E., and Fenwick, P. (1995). The truth in the light: An investigation of over 300 near-death experiences. London, England: Hodder Headline.

Gastaut, H. (1978). Fyodor Mikhailovitch Dostoevsky's involuntary contribution to the symptomatology and prognosis of epilepsy (trans. by R. Broughton). Epilepsia, 19, 186-201.

Gowers, W. R. (1881). Epilepsy and other chronic convulsive diseases: Their causes, symptoms, and treatment. London, England: Churchill.

Hameroff, S. R. (1987). Ultimate computing: Biomolecular consciousness and nano technology. New York, NY: Elsevier.

Jahn, R. G., and Dunne, B. J. (1987). Margins of reality: The role of consciousness in the physical world. San Diego, CA: Harcourt, Brace, Jovanovich.

Kuhn, T. S. (1970). The structure of scientific revolutions (2nd ed.). Chicago, IL: University of Chicago Press.

Jones-Gotman, M. (1987). Commentary: Psychological evaluation, testing hippocampal function. In J. Engel (Ed.), Surgical treatment of the epilepsies (pp. 203-211). New York, NY: Raven Press.

Lennox, W. G. (1960). Epilepsy and related disorders. Boston, MA: Little, Brown.

Ojemann, G. A., and Engel, J. Acute and chronic intracranial recording and stimulation. In J. Engel (Ed.), Surgical treatment of the epilepsies (pp. 263-288). New York, NY: Raven Press.

Penfield, W., and Kristiansen, K (1951). Epileptic seizure patterns. Springfield, IL: Charles C Thomas.

Penrose, R. (1994). Shadows of the mind: On consciousness, computation, and the new physics of the mind. New York, NY: Oxford University Press.

Saavedra-Aguilar, J. C., and Gómez-Jeria, J. S. (1989). A neurobiological model for near-death experiences. Journal of Near Death Studies, 7, 205-222. 
Teasdale, G. (1991). Head injury: Concussion, coma and recovery from altered states of consciousness. In M. Swash and J. Oxbury (Eds.), Clinical neurology (pp. 684-686). Edinburgh, Scotland: Churchill Livingstone.

Velmans, M. (1991). Consciousness from a first-person perspective. Behavioral and Brain Sciences, 14, 702-726.

Velmans, M. (1993). Consciousness, causality and complementarity. Behavioral and Brain Sciences, 16, 409-416.

Williamson, P. D., Wieser, H.-G., and Delgado-Escueta, A. V. (1987). Clinical characteristics of partial seizures. In J. Engel (Ed.), Surgical treatment of the epilepsies (pp. 101-120). New York, NY: Raven Press.

Wolman, B. B. (Ed.). (1977). Handbook of parapsychology. New York, NY: Van Nostrand Reinhold. 